\title{
Performance Evaluation of Biozentech Malaria Scanner in Plasmodium knowlesi and $P$. falciparum as a New Diagnostic Tool
}

\author{
Egy Rahman Firdaus ${ }^{1}$, Ji-Hoon Park', Fauzi Muh', Seong-Kyun Lee', Jin-Hee Han ${ }^{1}$, Chae-Seung Lim² ${ }^{10}$, \\ Sung-Hun $\mathrm{Na}^{3}$, Won Sun Park', Jeong-Hyun Park ${ }^{5}$, Eun-Taek Han ${ }^{1, *}$ (B) \\ 'Department of Medical Environmental Biology and Tropical Medicine, School of Medicine, Kangwon National University, Chuncheon 24341, Korea; \\ 2Department of Laboratory Medicine, Korea University College of Medicine, Seoul 08308, Korea; ${ }^{3}$ Department of Obstetrics and Gynecology, \\ Kangwon National University Hospital, Kangwon National University School of Medicine, Chuncheon 24341, Korea; ${ }^{4}$ Department of Physiology, \\ School of Medicine, Kangwon National University, Chuncheon 24341, Korea; ${ }^{5}$ Department of Anatomy and Cell Biology, School of Medicine, \\ Kangwon National University, Chuncheon 24341, Korea
}

\begin{abstract}
The computer vision diagnostic approach currently generates several malaria diagnostic tools. It enhances the accessible and straightforward diagnostics that necessary for clinics and health centers in malaria-endemic areas. A new computer malaria diagnostics tool called the malaria scanner was used to investigate living malaria parasites with easy sample preparation, fast and user-friendly. The cultured Plasmodium parasites were used to confirm the sensitivity of this technique then compared to fluorescence-activated cell sorting (FACS) analysis and light microscopic examination. The measured percentage of parasitemia by the malaria scanner revealed higher precision than microscopy and was similar to FACS. The coefficients of variation of this technique were $1.2-6.7 \%$ for Plasmodium knowlesi and $0.3-4.8 \%$ for $P$. falciparum. It allowed determining parasitemia levels of $0.1 \%$ or higher, with coefficient of variation smaller than $10 \%$. In terms of the precision range of parasitemia, both high and low ranges showed similar precision results. Pearson's correlation test was used to evaluate the correlation data coming from all methods. A strong correlation of measured parasitemia $\left(r^{2}=0.99, P<0.05\right)$ was observed between each method. The parasitemia analysis using this new diagnostic tool needs technical improvement, particularly in the differentiation of malaria species.
\end{abstract}

Key words: Plasmodium knowlesi, P. falciparum, parasitemia, malaria, diagnosis, computer vision

\section{INTRODUCTION}

Malaria is a life-threatening disease and a major cause of death worldwide. This disease is still one of the top-ranked infectious diseases, following human immunodeficiency viruses and tuberculosis [1]. Over 91 countries responsible for 216 million cases were visited annually by more than 125 million international travelers. Malaria also kills a child every 2 min, with the number of deaths reaching 435,000 in 2017 [2].

Approximately 250 species of the genus Plasmodium can infect mammals, birds, and reptiles, and there are 6 species responsible for human infection [3]. Each species has different features, such

- Received 17 February 2021, revised 11 March 2021, accepted 11 March 2021

*Corresponding author (etaekhan@gmail.com)

(c) 2021. Korean Society for Parasitology and Tropical Medicine

This is an Open Access article distributed under the terms of the Creative Commons Attribution Non-Commercial License (https://creativecommons.org/licenses/by-nc/4.0) which permits unrestricted non-commercial use, distribution, and reproduction in any

medium, provided the original work is properly cited. as morphological appearance, signs, symptoms, and consequences following appropriate treatment to the patients [4]. Malaria is diagnosed with the patients' clinical signs, symptoms, and physical findings on examination. The symptoms found in non-acute malaria share similarities with other diseases and potentially lead to misdiagnosis.

Proper and rapid diagnosis is required to reduce the morbidity and mortality caused by malaria. In endemic areas, the detection of asymptomatic cases is still a challenge for blocking the source of transmission. In addition, in nonendemic areas, very low parasite density is commonly found in infected individuals. A highly sensitive test is crucial in detecting low parasite density that may develop into severe disease $[5,6]$. These issues make diagnostic development a major concern in malaria eradication and elimination attempts [7]. The new malaria diagnostics have continuously developed to achieve excellent performance that is affordable, sensitive, specific, user-friendly, rapid and robust, equipment-free, and can be delivered to various areas 
$[8,9]$. This study focuses on P. knowlesi, the fifth human malaria parasite. P. knowlesi infection has become an interesting subject over the past few years. P. knowlesi began to be considered a threat since the infection was found in Malaysia and began to spread across Southeast Asia to Indonesia, Thailand, Myanmar, Singapore, Philippines, Vietnam, Cambodia, and Laos $[10,11]$. On the other hand, the clinical disease from $P$. knowlesi infection shows lower severity than $P$. falciparum infection, but higher severity than infection with those of other malaria parasite species [12]. However, the diagnosis of $P$. knowlesi is still inadequate enough to be studied. The easiest way to detect $P$. knowlesi is by microscopy. However, it should have a differential diagnosis from other human malaria parasites. Misdiagnoses that occurred in P. knowlesi which was identified as P. malariae or P. falciparum, led to a fatal outcome.

Other malaria diagnostic tools, such as rapid diagnostic tests (RDTs), has lower sensitivity than molecular tests in detecting P. knowlesi, especially at low parasitemia. Also, the inconsistency of RDTs among developers is another issue when applying them as a $P$. knowlesi detection method $[11,13]$. Immunofluorescence assay (IFA), enzyme-linked immunosorbent assay (ELISA), and fluorescence-activated cell sorting (FACS) analysis are considered to have a high sensitivity. However, they are relatively complicated and costly techniques [14,15]. Most of the diagnosis methods require fixation before analysis, and many reports described that fixation using methanol or heat inactivation would negatively affect the outcome of examination $[16,17]$

To overcome the limitations of current malaria diagnostics, automated microscopy based on computer vision techniques has been developed, and some of them are commercially available. These techniques provide consistency, and accurate diagnosis without the expert needed [18]. Our approach concerned to develop diagnostic techniques that provide malaria detection in a broad spectrum, from the laboratory to the endemic $P$. knowlesi malaria areas. A novel malaria diagnostic system using a new computer vision called the Biozentech malaria scanner was evaluated in this study. This system is developed by Biozentech, a subsidiary of Korea University Guro Hospital, considered simple, low cost, user-friendly, and highly sensitive. The size of the machine could be easily transferred to various areas, indicating that it was suitable for use in the clinical field.

This study was conducted to evaluate the performance of the Biozentech malaria scanner in in vitro detection of $P$. knowlesi and $P$. falciparum parasites. This method has been developed for clinical and laboratory purposes. This technique was given a proper analysis of $0.1 \%$ parasitemia or above. In addition, the high range of parasitemia had a similar precision with a low range. In terms of compression, the measured percentage of parasitemia by malaria scanner revealed similarity to microscopy and FACS; however, it has shown different accuracy. The malaria scanner has relatively similar accuracy to FACS and was better than light microscopy. Besides, this technique also has a strong correlation with microscopy and FACS. This study supports malaria eradication, particularly in the development of malaria diagnostics based on a computerized system.

\section{MATERIALS AND METHODS}

In vitro cultivation of $P$. knowlesi $\mathrm{A} 1-\mathrm{H} 1(\mathrm{PkA} 1 \mathrm{H} 1)$ and

\section{P. falciparum 3D7 (Pf3D7)}

This study used PkA1H1 parasite, a kind gift from Robert W. Moon, LSHTM. The detail protocol described elsewhere [19]. We used the same method for culturing Pf3D7 strain parasite with slightly different supplementation concentrations: 100 uM L-glutamine (Gibco, Paisley, Scotland), sodium bicarbonate (Sigma-Aldrich, St. Louis, Missouru, USA), hypoxanthine (Sigma-Aldrich), and 0.5\% albumax I (Gibco, Auckland, New Zealand). Both parasites were cultured at $2 \%$ hematocrit with mixed gases $\left(90 \% \mathrm{~N}_{2}, 5 \% \mathrm{CO}_{2}\right.$, and $\left.5 \% \mathrm{O}_{2}\right)$.

\section{Sample preparation}

A serial parasitemia was set at $10,5,1,0.5$, and $0.1 \%$ to compare sensitivity and reproducibility of the malaria scanner to microscopy and FACS. Two percent hematocrit cultured parasite was serially diluted by $1: 1$ using $2 \%$ hematocrit of healthy RBCs. A couple of ranges of parasitemia were defined as high- and low-range parasitemia. It was set to know the detection limit of the malaria scanner. The high range of parasitemia was set $5.0-0.3 \%$, while the low range was $1.0-0.1 \%$. Each parasite was aliquoted and examined in triplicate by microscopy, FACS, and malaria scanner.

\section{Detection of malaria parasites by microscopic examination}

Approximately $200 \mu \mathrm{l}$ of parasite culture was centrifuged, and its supernatant was discarded. The thin blood smear was made by spotting $2 \mu \mathrm{l}$ of culture pack cells onto a glass slide, fixed using 100\% ethanol, and then applying 10\% Giemsa 
staining solution for $10 \mathrm{~min}$. Parasitemia was counted using light microscopy at 1,000 $\times$ magnification for 20,000 red blood cells (RBCs).

\section{Malaria parasite detection using a scanner}

The machine algorithm allowed to classified cells based on their size and fluorescence intensity. Several parameters (size, dilution ratio, intensity, and gain) had been optimized in the prior, resulting in proper detection (Fig. 1). Plasmodium knowlesi A1-H1 culture was diluted 500 times from RBC pack cells using phosphate-buffered saline (PBS). This diluted sample was then mixed with $10 \times$ SYBR green I at a ratio of 1:1.5 from the total volume. Then, this mixture was placed in a slide chamber, incubated for $10 \mathrm{~min}$ until the culture was stable, and shuttled down on the surface chamber without any movements. The slide chamber was scanned automatically with a slight adjustment after using an autofocused option.

\section{Malaria parasite detection by FACS analysis}

To evaluate parasitemia counting analysis of the malaria scanner, a couple of sets of culture PkA1H1 and Pf3D7 parasites were detected and compared with the FACS technique. The parasite was fixed with $0.05 \%$ glutaraldehyde following incubation for $10 \mathrm{~min}$ at RT. The fixed parasite was then treat- ed with 1:10,000 of SYBR green I. PBS was used for washing before and after applying the dye and for diluting the parasite sample. The sample was examined at 20,000 events of each sample 5 times. The best 3 of the data were chosen for further analysis.

\section{Statistical analysis}

The sensitivity and reproducibility of the malaria scanner were analyzed using the standard regression method (GraphPad Software, San Diego, California, USA). The coefficient of variation was calculated by dividing the standard deviation by the mean. Pearson's correlation test was used to evaluate correlation data coming from all methods

\section{RESULTS}

The malaria scanner optimally detected 20 fields from a maximum of 30 fields, each field effectively detected 1,000 cells. The count encountered an error when more than 1,000 cells per field were detected. It was because the overlapping cells were more difficult to focus on resulting data analysis which was unstable and took longer.

To determine the sensitivity and detection limit of the malaria scanner, we identified 2 different ranges of parasitemia.
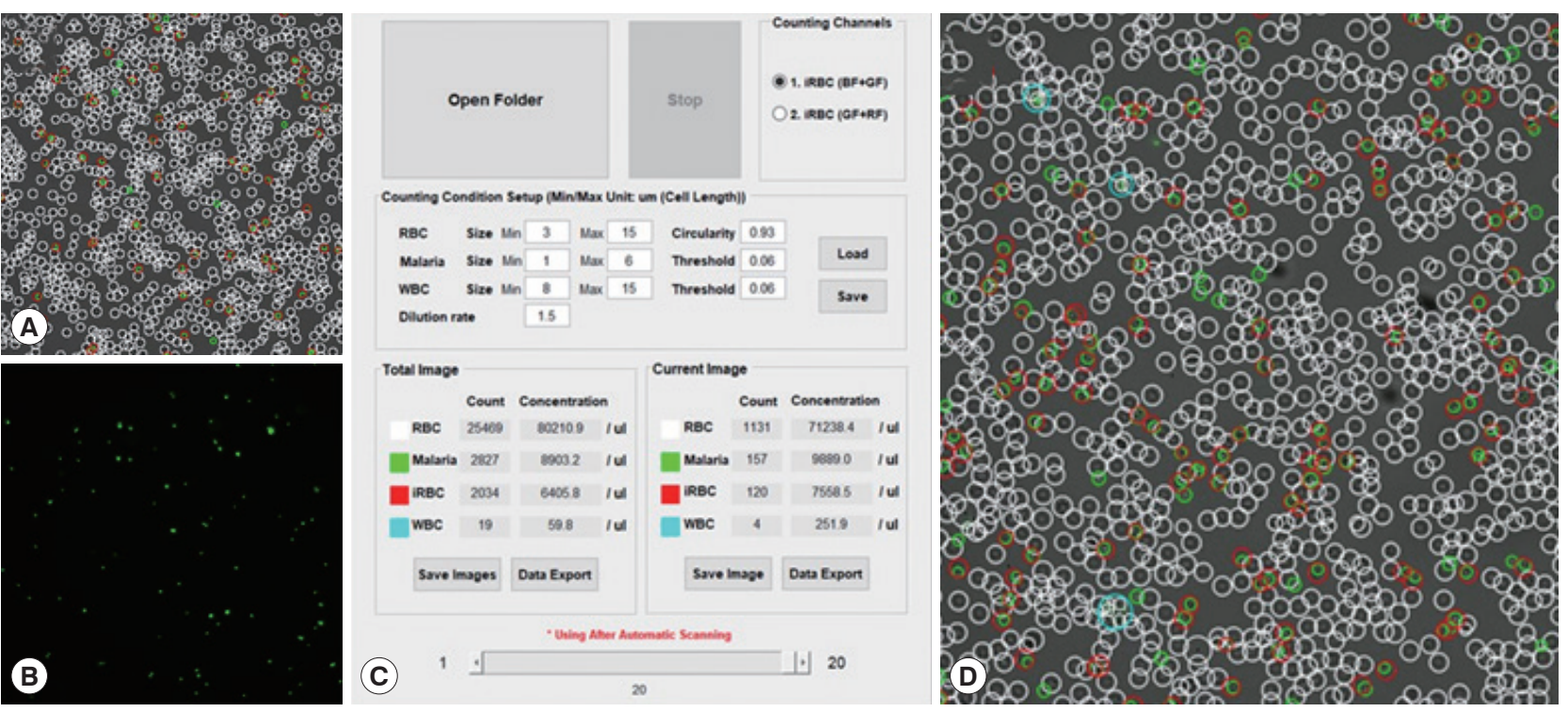

Fig. 1. Field and parameters of malaria scanner display. (A) Bright field, (B) green field, (C) parameters, and (D) merge field. A bright field described a field in DIC images. A green field was observed after applying SYBR green I. The parameters consisted of a size of each object, red blood cell, (RBC), free merozoites (Malaria), white blood cell (WBC), RBC circularity, threshold intensity (values) and infected $\mathrm{RBC}$ (iRBC). 


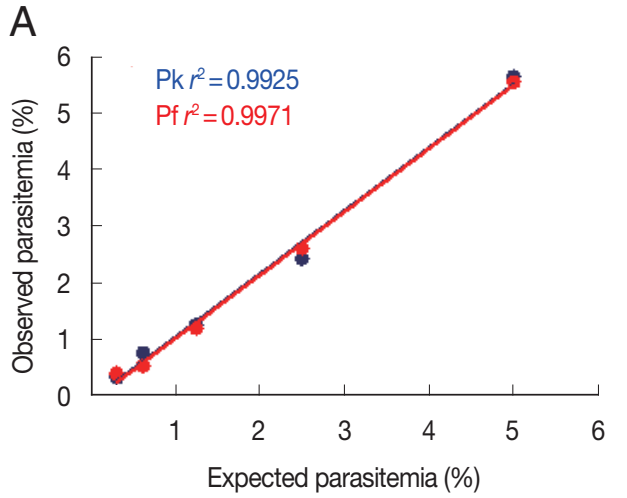

C

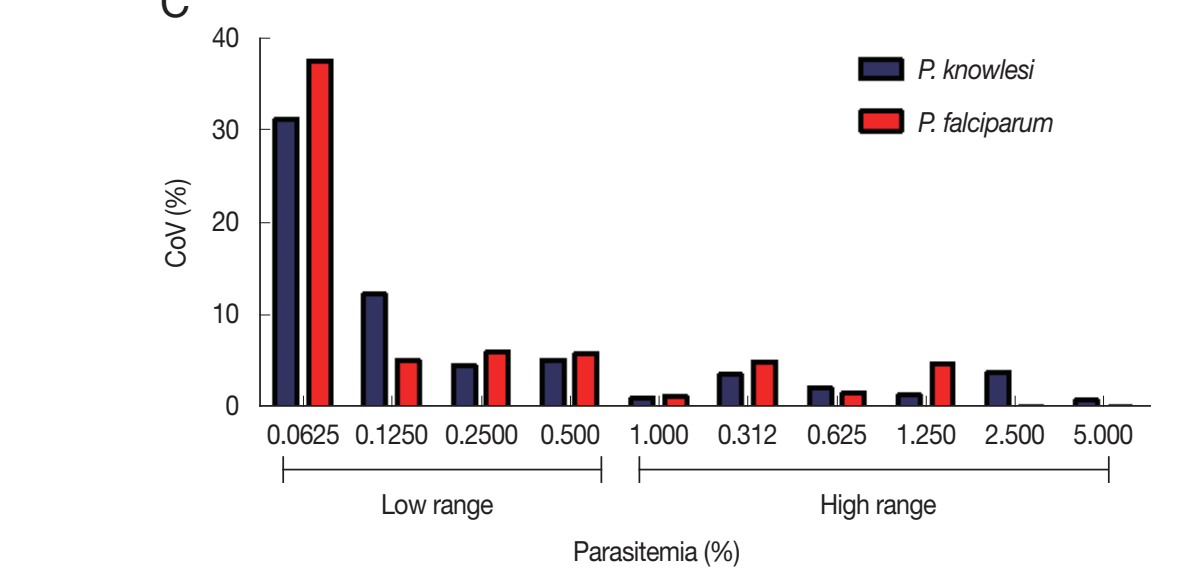

B

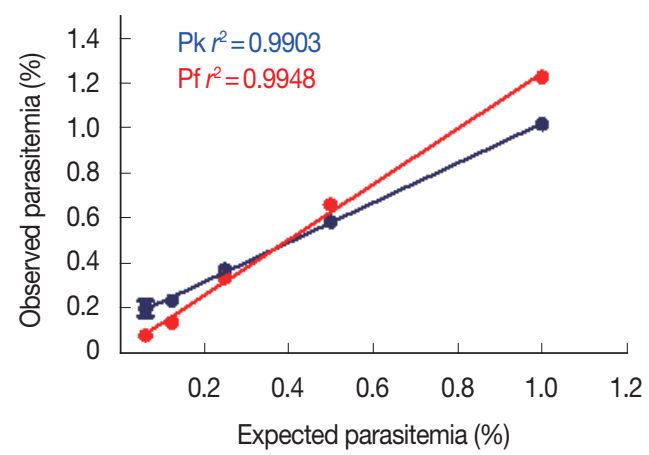

Fig. 2. Parasite detection in high and low parasitemia. Correlation between the expected and observed parasitemia in the high (A) and low (B) parasitemia, and coefficient of variation of each sample dilution in the high (C) and low (D) parasitemia.

The sensitivity of high-range parasitemia was relatively similar to the low-range for both P. knowlesi and P. falciparum. Both ranged show a similar correlation between expected and the measured parasitemia. The $r^{2}$ values from both ranges of parasitemia were 0.9925 and 0.9971 , respectively for $P$. knowlesi and $P$. falciparum in the high range. Meanwhile, the low range of parasitemia showed 0.9903 and 0.9948 , respectively for $P$. knowlesi and P. falciparum (Fig. 2A, B). Based on the coefficient of variation $(\mathrm{CoV})$, the malaria scanner allowed us to determine the parasite count in $0.1 \%$ or higher parasitemia for both $P$. knowlesi and P. falciparum (CoV 10) (Fig. 2C, D). The data showed that $0.06 \%$ of parasitemia has a $\mathrm{CoV}$ greater than 30 , which was not considered an acceptable value $(\mathrm{CoV}<10)$. Moreover, parasitemia below $0.1 \%$ gave a measurement error that detected higher parasitemia than the actual number (data not shown).

Performance evaluation of the malaria scanner was also conducted by comparing its parasitemia with FACS and microscopy. The malaria scanner has higher accuracy than mi- croscopy, but it was similar to FACS. Table 1 described $P$. knowlesi and P. falciparum by looking at the CoV of each technique. The $\mathrm{CoV}$ of the malaria scanner was $1.2-6.7 \%$ and $0.3-$ $4.8 \%$, and the FACS was $1.6-11.8 \%$ and $1.6-6.4 \%$ for $P$. knowlesi and $P$. falciparum, respectively. The CoV of microscope was 0.04-28.5\% and 0.1-23.1\%, respectively for P. knowlesi and $P$. falciparum. The percentage of parasitemia from all methods showed a strong correlation, as analyzed by Pearson's correlation tests $\left(r^{2}=0.99, P<0.05\right)$.

\section{DISCUSSION}

Computer vision plays a significant role in the healthcare sector, in particular for diagnosis of diseases. Excellent accura$\mathrm{cy}$, reproducibility, and cost efficiency are the reasons why computer vision diagnostics should be more developed. Computer vision can exploit morphological objects such as texture, shape, and contour. It allows comparing our results with prior knowledge with contextual information relay to the image se- 
Table 1. Comparison of Plasmodium knowlesi and $P$. falciparum parasitemia using light microscopy, FACS, and malaria scanner

\begin{tabular}{|c|c|c|c|c|c|c|c|c|c|}
\hline \multirow{2}{*}{ Expected parasitemia (\%) } & \multicolumn{3}{|c|}{ Light microscopy } & \multicolumn{3}{|c|}{ FACS } & \multicolumn{3}{|c|}{ Malaria scanner } \\
\hline & Mean & $\mathrm{SD}^{*}$ & $\mathrm{CoV}^{\star \star}(\%)$ & Mean & $\mathrm{SD}$ & $\operatorname{CoV}(\%)$ & Mean & SD & $\operatorname{CoV}(\%)$ \\
\hline \multicolumn{10}{|l|}{ Plasmodium knowlesi } \\
\hline 10 & 10.50 & 0.004 & 0.04 & 8.78 & 0.14 & 1.6 & 9.48 & 0.12 & 1.2 \\
\hline 5 & 5.08 & 0.07 & 1.3 & 4.10 & 0.05 & 1.3 & 4.86 & 0.10 & 2.1 \\
\hline 1 & 0.88 & 0.09 & 9.9 & 0.72 & 0.02 & 3.7 & 0.84 & 0.02 & 2.1 \\
\hline 0.5 & 0.48 & 0.06 & 12.1 & 0.48 & 0.02 & 3.3 & 0.47 & 0.02 & 6.1 \\
\hline 0.1 & 0.19 & 0.06 & 28.5 & 0.08 & 0.01 & 11.8 & 0.10 & 0.01 & 6.7 \\
\hline \multicolumn{10}{|l|}{ Plasmodium falciparum } \\
\hline 10 & 10.15 & 0.01 & 0.1 & 9.73 & 0.16 & 1.6 & 9.63 & 0.03 & 0.3 \\
\hline 5 & 4.52 & 0.18 & 4.0 & 4.30 & 0.09 & 2.0 & 5.40 & 0.04 & 0.8 \\
\hline 1 & 1.38 & 0.36 & 25.8 & 1.14 & 0.02 & 1.8 & 1.06 & 0.00 & 0.3 \\
\hline 0.5 & 0.65 & 0.13 & 19.3 & 0.45 & 0.03 & 6.3 & 0.52 & 0.01 & 0.9 \\
\hline 0.1 & 0.13 & 0.03 & 23.1 & 0.23 & 0.02 & 6.4 & 0.11 & 0.01 & 4.8 \\
\hline
\end{tabular}

*SD, Standard deviation; ${ }^{* *} \mathrm{CoV}$, Coefficient of variation.

quence, which is helpful in the diagnostic process [20].

Several diagnostic platforms have been created based on computer vision systems for tuberculosis, cancer, and malaria. In terms of malaria, there are several automated microscopy computer vision systems, such as World Health Technology (WHT) automated scanning, autoscope, and automated diagnostic applications [21,22]. To improve the accuracy from wide variations of slide-to-slide and examiner skills, computer malaria diagnostics have been continuously evaluated using clinical samples $[23,24]$.

The analysis used an algorithm that mainly works with object identification following object differentiation between several items belonging to the same group (grouping). Short detection analysis was provided by focusing on the surface of the live cells in both infected and uninfected malaria parasites. It could differentiate parasite-infected red blood cells from other cells based on the size and fluorescence intensity of each cell.

Simple preparation without sample fixation leads to faster analysis and reduces the possibility of sample changes or even damage that may produce inaccurate data. This method only required dilution of the sample and application of a dye reagent. We used SYBR Green I, which is known to distinguish cells in a broad application $[25,26]$. In addition, a malaria scanner was designed as user-friendly and more accessible in the field, providing analysis in clinical malaria-endemic areas in the future.

Plasmodium knowlesi and P. falciparum could be detected well in both high and low ranges of parasitemia by the malaria scanner with higher sensitivity compared to microscopy. However, the method sensitivity relatively similar compared to
FACS. The subjectivity of the microscope analysis and limited observed fields resulted in substantial variation [27]. Besides, parasitemia counting by FACS was relatively less than microscope and malaria scanner. This application was probably due to sample preparation, particularly during sample dilution, fixation, or dye staining processes that caused cell lysis and morphological changes.

A limitation of this method has used a sample from in vitro cultured parasite, it comes to possibilities that the result might differ from the clinical sample. Moreover, this analysis used a living parasite, which leads to RBC dehydration, having a consequence on a single-use sample. Dehydrated parasites have morphological alterations, resulting in improper analysis during extended periods [28]. The sensitivity is slightly affected by dilution and incubation time during the examination. The sample must be incubated for at least 8-10 min to ensure it properly stayed on the chamber or would not affect the image focus. Moreover, this method is still not able to distinguish different species of parasites. Thus, crosschecking by Giemsa staining is still needed. In image technique-based quantification analysis, the focusing step on parasite images is essential during examination [29]. Incubation time also slightly affected the fluorescence intensity that was produced from the DNA content in the sample [25].

In the end, this study supported the development of a computer malaria diagnostic system for $P$. knowlesi. Differentiation of malaria parasite species is required to improve this technique. Additionally, an evaluation of the current tool in the clinical sample is essential for clinical automation in malaria diagnostics. User-friendly, easy maintenance, fast and high 
sensitivity detection demonstrated that the malaria scanner could be potentially applied in a wide malaria research field, such as drug, antibodies, and vaccine efficiency evaluation.

\section{ACKNOWLEDGMENTS}

The authors are grateful to Robert W. Moon Department of Immunology and Infection, Faculty of Infectious and Tropical Diseases, London School of Hygiene and Tropical Medicine, London WC1E 7HT, United Kingdom for providing the P. knowlesi A1-H.1 strain. This study was supported by a grant from the Korea Association of Health Promotion (2019-02), the National Research Foundation of Korea (NRF) grant funded by the Korea government (MSIP) (NRF-2017R1A2A2A05069562), by Basic Science Research Programmed through the National Research Foundation of Korea (NRF) funded by the Ministry of Science, ICT and Future Planning (2015R1A4A1038666). The funders had no role in study design, data collection, and analysis, decision to publish, or preparation of the manuscript.

\section{CONFLICT OF INTEREST}

The authors declare that they have no conflict of interest.

\section{REFERENCES}

1. Pigott DM, Howes RE, Wiebe A, Battle KE, Golding N, Gething PW, Dowell SF, Farag TH, Garcia AJ, Kimball AM. Prioritising infectious disease mapping. PLoS Negl Trop Dis 2015; 9: e0003756. https://doi.org/10.1371/journal.pntd.0003756

2. Parselia E, Kontoes C, Tsouni A, Hadjichristodoulou C, Kioutsioukis I, Magiorkinis G, Stilianakis NI. Satellite earth observation data in epidemiological modeling of malaria, dengue and West Nile virus: a scoping review. Remote Sens 2019; 11: 1862. https://doi.org/10.3390/rs11161862

3. Ramasamy R. Zoonotic malaria-global overview and research and policy needs. Front Public Health 2014; 2: 123. https://doi. org/10.3389/fpubh.2014.00123

4. Ortiz-Ruiz A, Postigo M, Gil-Casanova S, Cuadrado D, Bautista JM, Rubio JM, Luengo-Oroz M, Linares M. Plasmodium species differentiation by non-expert on-line volunteers for remote malaria field diagnosis. Malar J 2018; 17: 54. https://doi.org/10.1186/ s12936-018-2194-8

5. Wiese L, Bruun B, Bæk L, Friis-Møller A, Gahrn-Hansen B, Hansen J, Heltberg O, Højbjerg T, Kathrine Hornstrup M, Kvinesdal B. Bedside diagnosis of imported malaria using the Binax Now malaria antigen detection test. Scand J Infect Dis 2006; 38: 10631068. https://doi.org/10.1080/00365540600818011
6. Alvar J, Alves F, Bucheton B, Burrows L, Büscher P, Carrillo E, Felger I, Hübner MP, Moreno J, Pinazo M-J, Ribeiro I, Estani-SS, Specht S, Tarral A, Wourgaft NS, Bilbe G. Implications of asymptomatic infection for the natural history of selected parasitic tropical diseases. Semin Immunopathol 2020; 42: 231-246. https://doi.org/10.1007/s00281-020-00796-y

7. Bronzan RN, McMorrow ML, Kachur SP. Diagnosis of malaria: challenges for clinicians in endemic and non-endemic regions. Mol Diagn Ther 2008; 12: 299-306. https://doi.org/10.1007/ BF03256295

8. Peeling RW, Holmes KK, Mabey D, Ronald A. Rapid tests for sexually transmitted infections (STIs): the way forward. Sex Transm Infect 2006; 82 (suppl): 1-6. http://dx.doi.org/10.1136/ sti.2006.024265

9. Kosack CS, Page AL, Klatser PR. A guide to aid the selection of diagnostic tests. Bull World Health Organ 2017; 95: 639-645. https://doi.org/10.2471/BLT.16.187468

10. Zaw MT, Lin Z. Human Plasmodium knowlesi infections in SouthEast Asian countries. J Microbiol Immunol Infect 2019; 52: 679684. https://doi.org/10.1016/j.jmii.2019.05.012

11. Singh B, Daneshvar C. Human infections and detection of Plasmodium knowlesi. Clin Microbiol Rev 2013; 26: 165-184. https:// doi.org/10.1128/cmr.00079-12

12. Cox-Singh J, Hiu J, Lucas SB, Divis PC, Zulkarnaen M, Chandran $P$, Wong KT, Adem P, Zaki SR, Singh B. Severe malaria-a case of fatal Plasmodium knowlesi infection with post-mortem findings: a case report. Malar J 2010; 9: 10. https://doi.org/10.1186/1475-2875-910

13. Mahende C, Ngasala B, Lusingu J, Yong T-S, Lushino P, Lemnge M, Mmbando B, Premji Z. Performance of rapid diagnostic test, blood-film microscopy and PCR for the diagnosis of malaria infection among febrile children from Korogwe District, Tanzania. Malar J 2016; 15: 391. https://doi.org/10.1186/s12936-016-1450-z

14. Spencer HC, Collins WE, Warren M, Jeffery GM, Mason J, Huong AY, Stanfill PS, Skinner JC. The enzyme-linked immunosorbent assay (ELISA) for malaria. Am J Trop Med Hyg 1981; 30: 747-750. https://doi.org/10.4269/ajtmh.1981.30.747

15. She RC, Rawlins ML, Mohl R, Perkins SL, Hill HR, Litwin CM. Comparison of immunofluorescence antibody testing and two enzyme immunoassays in the serologic diagnosis of malaria. J Travel Med 2007; 14: 105-111. https://doi.org/10.1111/j.17088305.2006.00087.x

16. Cutts T, Cook B, Poliquin G, Strong J, Theriault S. Inactivating Zaire Ebolavirus in whole-blood thin smears used for malaria diagnosis. J Clin Microbiol 2016; 54: 1157-1159. http://dx.doi. org/10.1128/JCM.02960-15

17. Fernando SD, Ihalamulla RL, Wickremasinghe R, de Silva NL, Thilakarathne JH, Wijeyaratne P, Premaratne RG. Effects of modifying the World Health Organization standard operating procedures for malaria microscopy to improve surveillance in resource poor settings. Malar J 2014; 13: 98. https://doi.org/10.1186/14752875-13-98

18. Pollak JJ, Houri-Yafin A, Salpeter SJ. Computer vision malaria 
diagnostic systems-progress and prospects. Front Public Health 2017; 5: 219. https://doi.org/10.3389/fpubh.2017.00219

19. Moon RW, Hall J, Rangkuti F, Ho YS, Almond N, Mitchell GH, Pain A, Holder AA, Blackman MJ. Adaptation of the genetically tractable malaria pathogen Plasmodium knowlesi to continuous culture in human erythrocytes. Proc Natl Acad Sci USA 2013; 110: 531-536. https://doi.org/10.1073/pnas.1216457110

20. Chen $\mathrm{CH}$. An introduction to computer vision in medical imaging. In Chen $\mathrm{CH}$ ed, Computer Vision in Medical Imaging. Toh Tuck Link, Singapore. World scientific. 2014, pp 1-16.

21. Prescott WR, Jordan RG, Grobusch MP, Chinchilli VM, Kleinschmidt I, Borovsky J, Plaskow M, Torrez M, Mico M, Schwabe C. Performance of a malaria microscopy image analysis slide reading device. Malar J 2012; 11: 155. https://doi.org/10.1186/14752875-11-155

22. Delahunt CB, Mehanian C, Hu L, McGuire SK, Champlin CR, Horning MP, Wilson BK, Thompon CM. Automated microscopy and machine learning for expert-level malaria field diagnosis. IEEE. 2015: 393-399. https://doi.org/10.1109/GHTC.2015.7344002

23. Eshel Y, Houri-Yafin A, Benkuzari H, Lezmy N, Soni M, Charles M, Swaminathan J, Solomon H, Sampathkumar P, Premji Z. Evaluation of the Parasight platform for malaria diagnosis. J Clin Microbiol 2017; 55: 768-775. https://doi.org/10.1128/JCM.02155-16
24. Vink J, Laubscher M, Vlutters R, Silamut K, Maude R, Hasan M, De Haan G. An automatic vision-based malaria diagnosis system. J Microsc 2013; 250: 166-178. https://doi.org/10.1111/jmi.12032

25. Martens-Habbena W, Sass H. Sensitive determination of microbial growth by nucleic acid staining in aqueous suspension. Appl Environ Microbiol 2006; 72: 87-95. https://doi.org/10.1128/ aem.72.1.87-95.2006

26. Grimberg BT. Methodology and application of flow cytometry for investigation of human malaria parasites. J Immunol Methods 2011; 367: 1-16. https://doi.org/10.1016/j.jim.2011.01.015

27. Watson OJ, Sumner KM, Janko M, Goel V, Winskill P, Slater HC, Ghani A, Meshnick SR, Parr JB. False-negative malaria rapid diagnostic test results and their impact on community-based malaria surveys in sub-Saharan Africa. BMJ Glob Health 2019; 4: e001582. http://dx.doi.org/10.1136/bmjgh-2019-001582

28. Gallagher PG. Disorders of erythrocyte hydration. Blood 2017; 130: 2699-2708. https://doi.org/10.1182/blood-2017-04-590810

29. Yoon J, Kwon JA, Yoon SY, Jang WS, Yang DJ, Nam J, Lim CS. Diagnostic performance of CellaVision DM96 for Plasmodium vivax and Plasmodium falciparum screening in peripheral blood smears. Acta Trop 2019; 193: 7-11. https://doi.org/10.1016/j.actatropica.2019.02.009 
